\title{
Unravelling the Potential of Natural Products - Biological Profiling of Extracts and New Molecules
}

\author{
Matthias Hamburger*
}

\begin{abstract}
Natural products are highly useful as sources of drugs and as tools for fundamental biomedical research. Phytomedicines based on medicinal plants and herbal extracts are used worldwide. Using three examples from our lab, we have shown different approaches to explore the biological and pharmacological properties of herbal extracts and new natural product leads. Our emphasis has been on global effects and multiple rather than single targets, whereby cell- and mechanism-based in vitro assays, in vivo pharmacology, cell biology and transcriptional profiling were employed.
\end{abstract}

Keywords: Cimicifuga racemosa $\cdot$ Expression profiling $\cdot$ HPLC-based activity profiling $\cdot$ Isatis tinctoria Natural products $\cdot$ Paecilomyces militaris $\cdot$ Phytomedicines $\cdot$ Signalling pathways

\section{Introduction}

Substances of biogenic origin have been used by mankind since prehistoric times to treat or alleviate human disease. Over the past two centuries, natural products have paved the way for modern pharmacotherapy. The contribution of natural products to the recent drug inventory has been substantial. Out of the 877 small molecule New Chemical Entities (NCEs) approved over the period from 1981 to $2002,49 \%$ were directly or indirectly derived from natural products. About half of the 35 top selling drugs worldwide in the years 2000-2002

\footnotetext{
${ }^{*}$ Correspondence: Prof. Dr. M. Hamburger Institute of Pharmaceutical Biology

Department of Pharmaceutical Sciences

Pharmacenter

University of Basel

Klingelbergstrasse 50

$\mathrm{CH}-4056$ Basel

Tel.: +41612671425

Fax: +4161267 1474

E-Mail: matthias.hamburger@unibas.ch

www.unibas.ch
}

were natural product-derived or biologicals. A significant number of natural product-derived molecules are currently in clinical trials in therapeutic areas such as infectious, neurological, cardiovascular, metabolic, inflammatory and immunological diseases, and cancer [1].

Secondary metabolites are products of co-evolutive processes and have been selected to provide some competitive advantage for the producing organism. As we know today, these molecules often address unique biological targets. Given the highly conserved nature of many bio-macromolecules and the limited number of protein families and structural domains on the one hand, and the high number ( $>200$ '000 secondary metabolites have been described up to now), structural diversity and complexity of natural products on the other, one may reasonably assume that some natural product ligand exists for a majority if not all proteins [2]. Natural products have been decisive for the progress of pharmacology, and numerous fundamental biological processes have been discovered and explored with the aid of these molecules [3]. Catalogues with research biochemicals are full of natural products possessing unique mechanisms of action.

There is, however, a second and quite different facet to drugs of biogenic origin. The World Health Organization (WHO) estimates that about $70 \%$ of the world population relies on medicinal plants as a primary source of drugs to treat their ail- ments. In industrialized countries, products containing herbal extracts are commercialized as phytopharmaceuticals or food supplements. There is convincing clinical evidence for the efficacy of phytomedicines such as Ginkgo biloba special extract, St. John's Wort (Hypericum perforatum), or saw palmetto (Serenoa repens) [4].

\section{Herbal Extracts - Multi- component Mixtures Addressing Multiple Pharmacological Targets}

Pharmaceutical products containing extracts as active ingredient are widely used in certain European countries such as Switzerland and Germany. Extracts are highly complex multi-component mixtures. A major argument put forward in favour of herbal extracts is that synergistic actions of compounds would confer a unique advantage to these preparations. However, convincing experimental demonstration of synergistic action is available in only a few cases, e.g. for the Ginkgo biloba special extract EGb761. The major obstacle has been a lack of knowledge on the active principles in the extract and on their molecular modes of action.

Some years ago, we decided to embark on a project to explore the issue of synergistic action in herbal extracts with stateof-the-art methodologies [5]. We selected woad (Isatis tinctoria L., Brassicaceae) as a model plant for this study. Woad is an old 
indigo dye plant of temperate climates, and a medicinal plant with a historical track record as an anti-inflammatory agent. A close botanical relative, Isatis indigotica FORT., is a major plant used in traditional Chinese medicine (TCM), also for the treatment of inflammatory ailments. Both species, however, lacked pharmacological evidence in support of their use.

As a first step, we confirmed the putative anti-inflammatory properties with an in vitro pharmacological profiling in a panel of $>20$ cell-based and mechanism-based assays addressing clinically relevant targets [5]. A promising pharmacological profile was observed using the dichloromethane extract from dry leaves (Fig. 1). An essential prerequisite for the intended study, namely a significant effect on a range of defined pharmacological targets, was hence satisfied.

We then identified compounds responsible for the individual facets of the extract's overall pharmacological profile with the aid of HPLC-based activity profiling [6]. For example, the COX-2 inhibitory activity could be traced to tryptanthrin, a minor alkaloid present in the extract (Fig. 2 ). The compound is a highly potent and isoenzyme-selective inhibitor of cyclooxygenase-2 (COX-2) ( $\mathrm{IC}_{50} 36 \mathrm{nM}$ in Mono Mac 6 cells). Tryptanthrin also strongly inhibits 5-lipoxygenase (5-LOX) and the expression of inducible nitric oxide synthase (iNOS) [7]. Other inhibitors of 5-LOX [8] and of leukocytic elastase (unpublished) have been identified. A minor benzylidenyl indolin-2-one derivative in the extract is a potent inhibitor of compound $48 / 80$ induced histamine release from rat mast cells and is, as such, significantly more active than the anti-asthmatic drug disodium chromoglycate [9].

In a collaboration with the group of Prof. José-Luis Rios, University of Valencia, we investigated the anti-inflammatory activity of tryptanthrin and Isatis extracts with in vivo pharmacological models of acute and subchronic inflammation [10]. In topical and oral applications, the extracts showed pronounced anti-inflammatory activity in the TPA-induced mouse ear oedema, whereas the effect of tryptanthrin was not statistically significant. In oral application, extracts were active in the carageenan-induced acute mouse paw oedema. In topical application, they also inhibited the delayed-type hypersensitivity reaction induced by dinitrofluorobenzene. A clinical pilot study with topical application of woad extracts and tryptanthrin showed that identical amounts of tryptanthrin applied as extract were superior to the pure compound in the prevention of experimentally-induced irritant contact dermatitis [11].

The superiority of woad extract over pure tryptanthrin in the animal and clini-

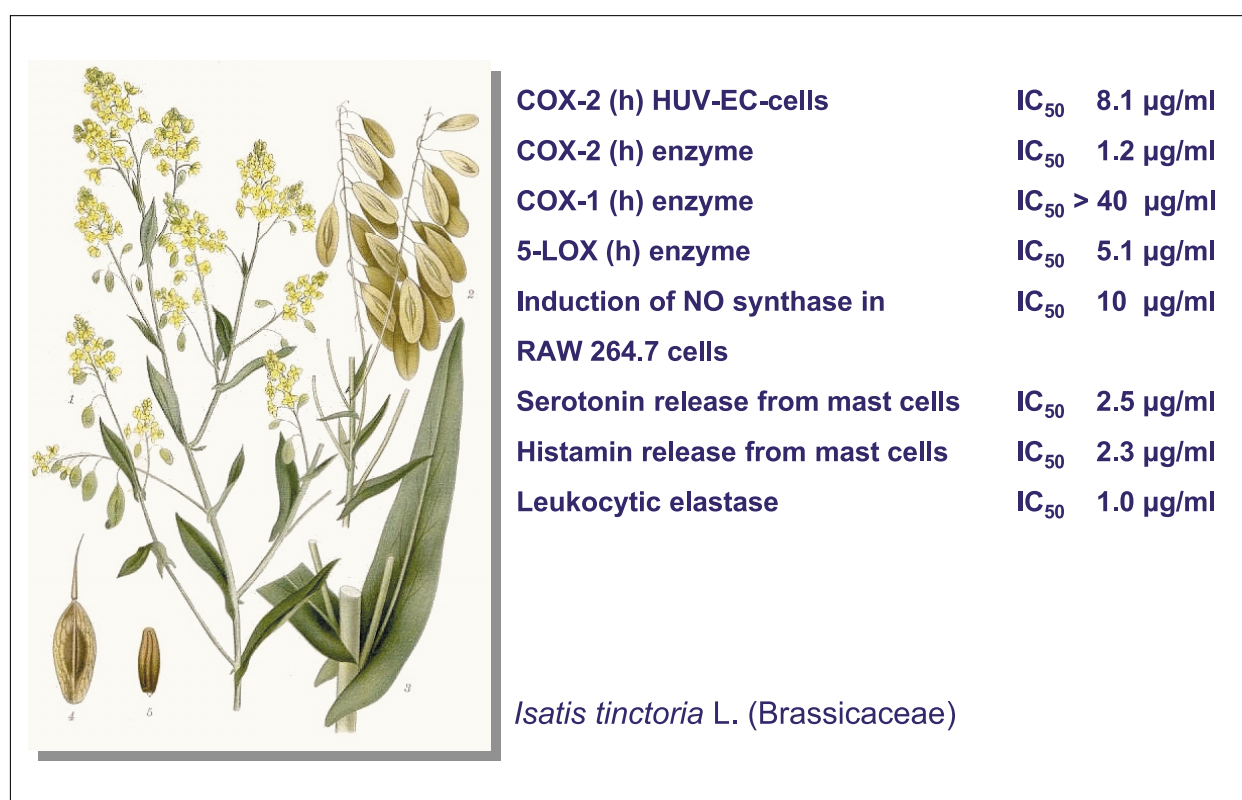

Fig. 1. In vitro pharmacological profile of a dichloromethane extract obtained from dried leaves; $(\mathrm{h})=$ human. The drawing of Isatis tinctoria depicts the biennial plant in the flowering stage (2nd year)
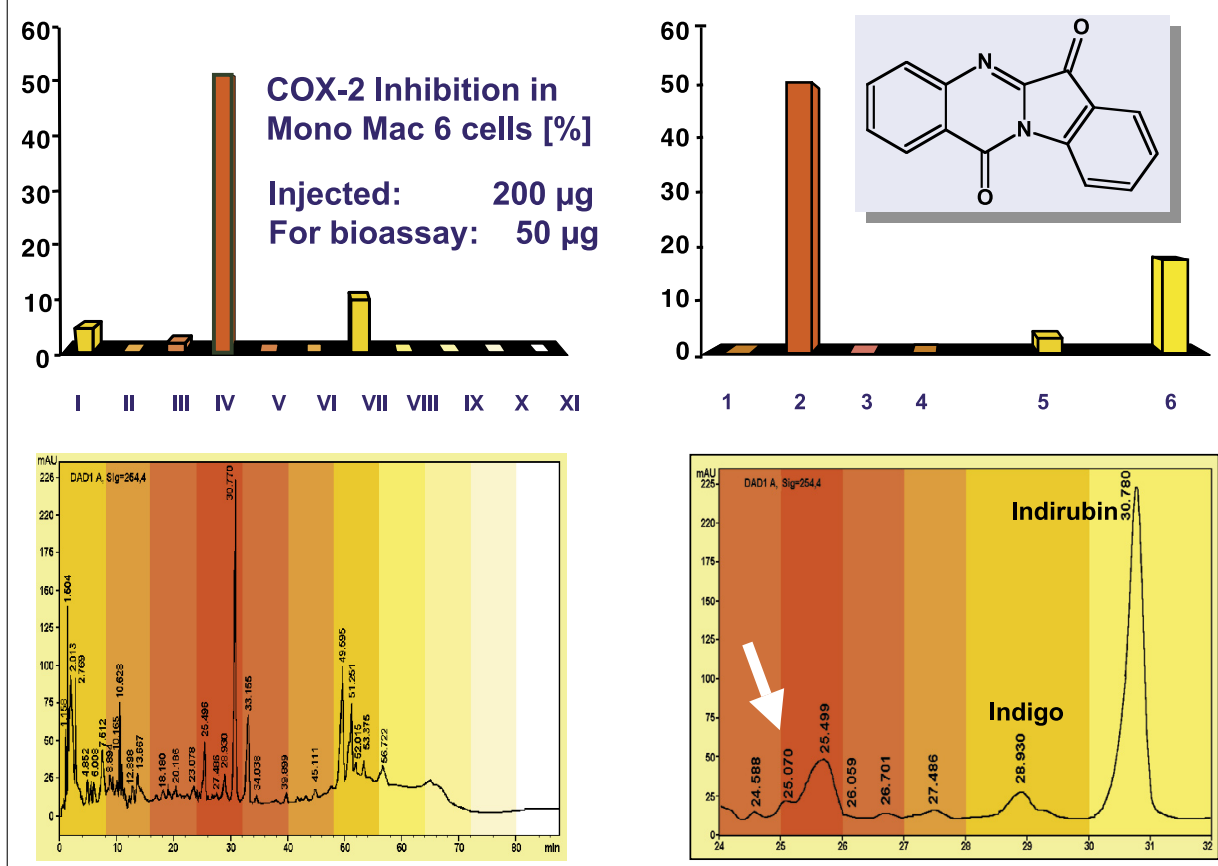

Fig. 2. HPLC-based activity profiling of /satis tinctoria. The gradient HPLC profile of the dichloromethane extract is shown below, and the inhibition profile on COX-2 catalyzed synthesis of 6-keto-PGF ${ }_{1 \alpha}$ in LPS-stimulated Mono Mac 6 cells of fractions corresponding to the colour-coded time windows is shown above. The left chromatogram corresponds to the extract, and the right chromatogram shows the time window of the active fraction IV at higher resolution. The arrow indicates the peak of the active compound tryptanthrin (structure in the inset).

cal models could have two reasons, namely synergistic effects with other pharmacologically active constituents in the extract, or improved bioavailability of the poorly soluble tryptanthrin due to the extract matrix. To address the latter point, we compared the skin penetration of pure tryptanthrin and tryptanthrin in extracts with the aid of cutaneous microdialysis coupled to stable isotope dilution LC-MS [12]. Compared to the pure substance, penetration of tryptanthrin from extract was dose-dependent and faster. Microscopic analysis of deposits showed that the alkaloid tends to crystallize 
as pure compound but remains molecularly disperse in the extract. We are currently performing a metabolite profiling of the plant to investigate the post-harvest formation of some of the pharmacologically active constituents [13]. The study of pharmacological synergy will subsequently be addressed with reconstituted compound mixtures of increasing complexity.

\section{New Natural Products - Exploring Their Mode of Action}

Natural products are important tools for basic research in the life sciences. Numerous biological processes have been deciphered, and pharmacological targets identified with the aid of secondary metabolites. Phenotypical screens provide global endpoints for complex biological events and are useful for the discovery of bioactive molecules which may act via unknown mechanisms. We have been using a phenotypical assay in the search for natural products with neurotrophic properties in an effort to discover new compounds acting on targets that are relevant in neurodegenerative diseases [14].

Prevention of neuronal apoptosis by supplementation with endogenous trophic factors has been proposed as one of several new strategies for the treatment of neurodegenerative disorders such as Alzheimer's disease. Clinical trials with neurotrophic factors, however, have been rather disappointing [15]. One reason for the failure was related to the obstacles in delivering therapeutic proteins to the central nervous system. The development of non-peptidic small molecules mimicking neurotrophin action has been proposed as an alternative [16].

With the aid of a phenotypical screen using PC12 cells, we recently identified fungal pyridone alkaloids as a new class of metabolites with neuritogenic properties. The most active compound, militarinone A, induced distinct neurite outgrowths at $10 \mu \mathrm{M}$ concentrations (Fig. 3) [17].

The PC12 cell line has been extensively used as a model for neuronal differentiation and neurotrophin action because of its ability to differentiate into sympathetic neuron-like cells when treated with nerve growth factor (NGF). The binding of NGF to its high-affinity tyrosine kinase A (TrkA) receptor triggers mainly two cascades of cellular signalling responses that mediate neurotrophic effects (Fig. 4) [18]. After binding to the phosphotyrosine-containing recognition elements of the receptor, signal molecules on the MAP kinase Erk1/2 and inositol triphosphate (PI3) kinase pathways are activated, resulting in effects on gene transcription and regulation of the cytoskeletal machinery. There is increasing evidence that another pathway involving SAPK/JNK

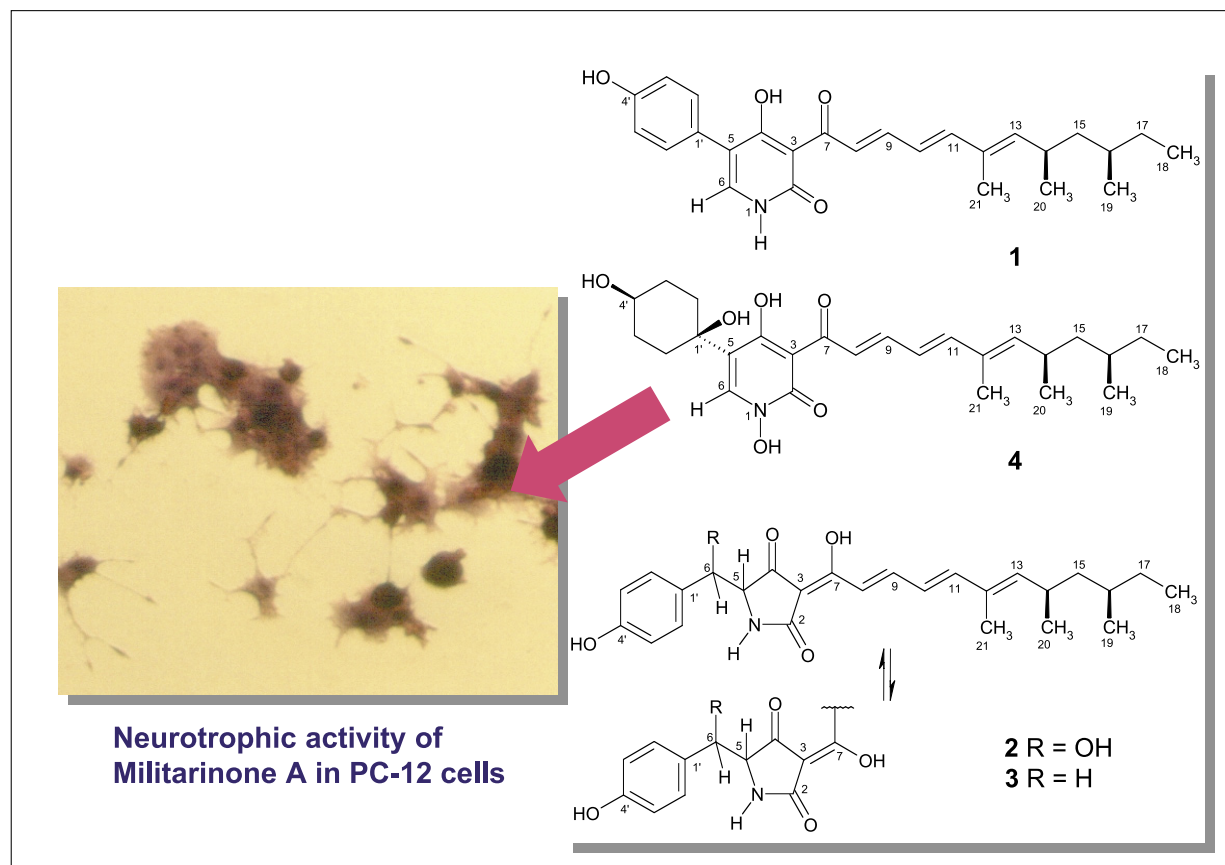

Fig. 3. Pyridone alkaloids and acyl tetramic acids from the insect pathogenic fungus Paecilomyces militaris RCEF 0095. The neurotrophic effect of militarinone A (4) in PC12 cells is shown in the inset (cells were stained for better contrast). Militarinone B (2), C (3) and D (1) show marginal neurotrophic properties. The acyl tetramic acids 2 and $\mathbf{3}$ are putative biosynthetic precurors of pyridone alkaloids.

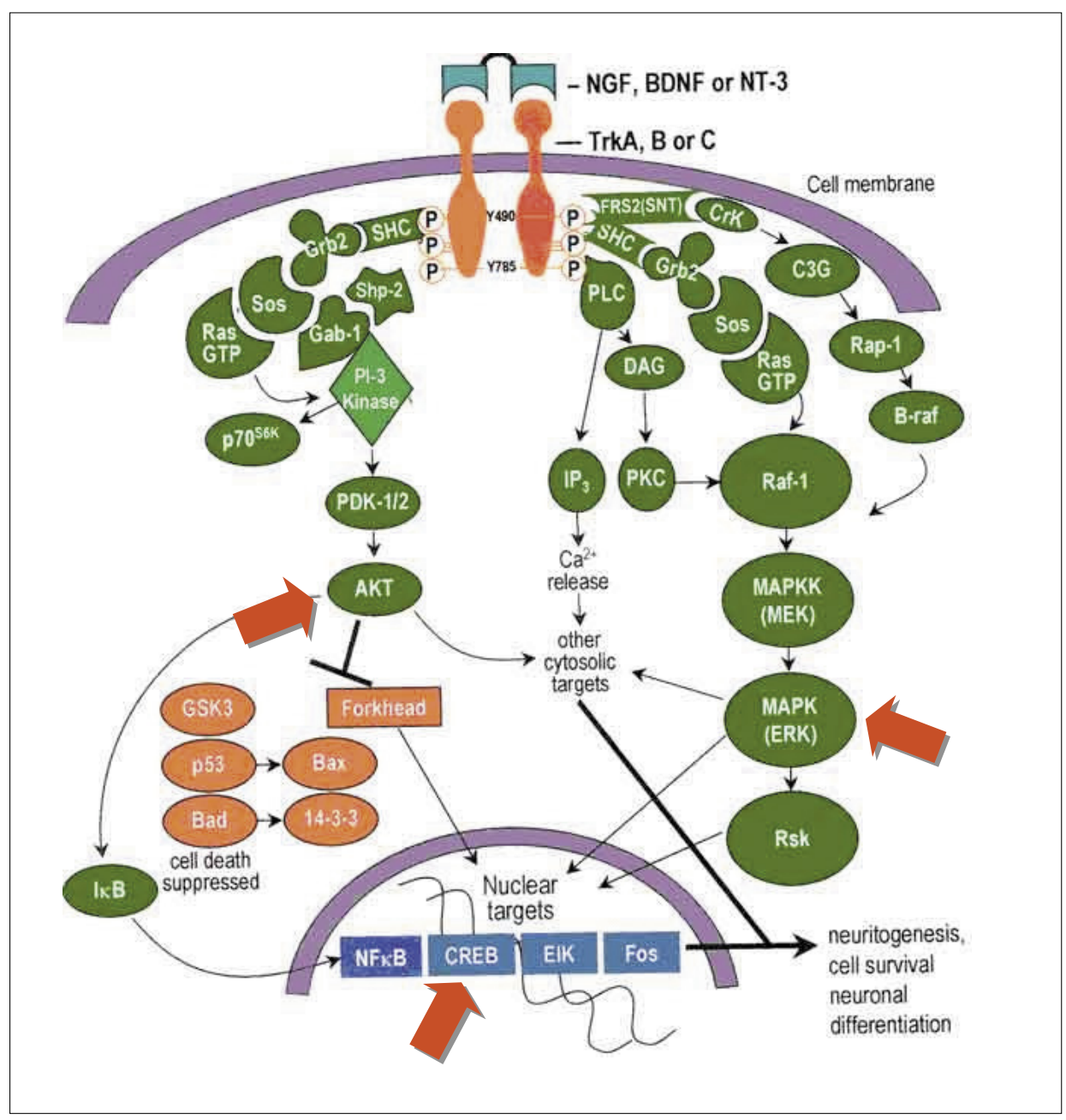

Fig. 4. Major signalling pathways for nerve growth factor (NGF). The red arrows relate to effects observed by treatment with militarinone A (4). 
has substantial influence on differentiation events. The transcription factor c-AMP-responsive element binding protein (CREB) appears to be required for NGF-mediated induction of primary response genes encoding transcription factors like c-fos, and thus plays a role in the initiation and regulation of subsequent responses to NGF. Phosphorylation of CREB is critical for learning processes, mainly in the conversion of short-term to long-term memory [19].

We analyzed the effects of militarinone A on the above-mentioned pathways to gain some insight into the mechanisms underlying the differentiation-inducing properties of this small molecule [20]. In primed cells, an early activation of protein kinase $\mathrm{B}(\mathrm{Akt})$, representing a downstream target of PI3-kinase, and a delayed phosphorylation of Erk1/2 and CREB were found. The NGF-dependent activation of SAP/JNK1 was potentiated. Morphological differentiation of cells and the phosphorylation of specific signal molecules were blocked by the MAP kinase inhibitor PD098059, the PI3-kinase inhibitor wortmannin, and the adenylyl cyclase inhibitor 9-cyclopentyladenine. We are currently studying the upstream events to identify the molecular target of militarinone A.

\section{Towards an Unbiased Assessment of Biological Profiles}

In recent years, a number of tools have become available which enable the study of global effects of molecules in biological systems [21]. A general trait of these systems-oriented methods is that they allow an essentially unbiased approach which does not need to be hypothesis-driven. Given that most natural products have been studied from a chemical viewpoint only, the potential of unravelling unexpected biological properties for these molecules via functional genomic and proteomic approaches is considerable. When extending such studies to herbal extract preparations, new insights into the overall effects of multi-component mixtures can be obtained. A seminal study in that respect was performed on the in vivo neuromodulatory effects of the standardized Ginkgo extract EGb761 [22].

With black cohosh (Cimicifuga racemosa L., Ranunculaceae) as a model, we are currently exploring the potential of genome-wide transcriptional profiling for the characterization of global effects of herbal extracts. Extracts from the rhizome of black cohosh are used as an alternative to hormone replacement therapy for the relief of symptoms in postmenopausal women. However, despite decades of clinical use, the molecular mode of ac-

\section{8 genes up/downregulated $>1.5$ fold}

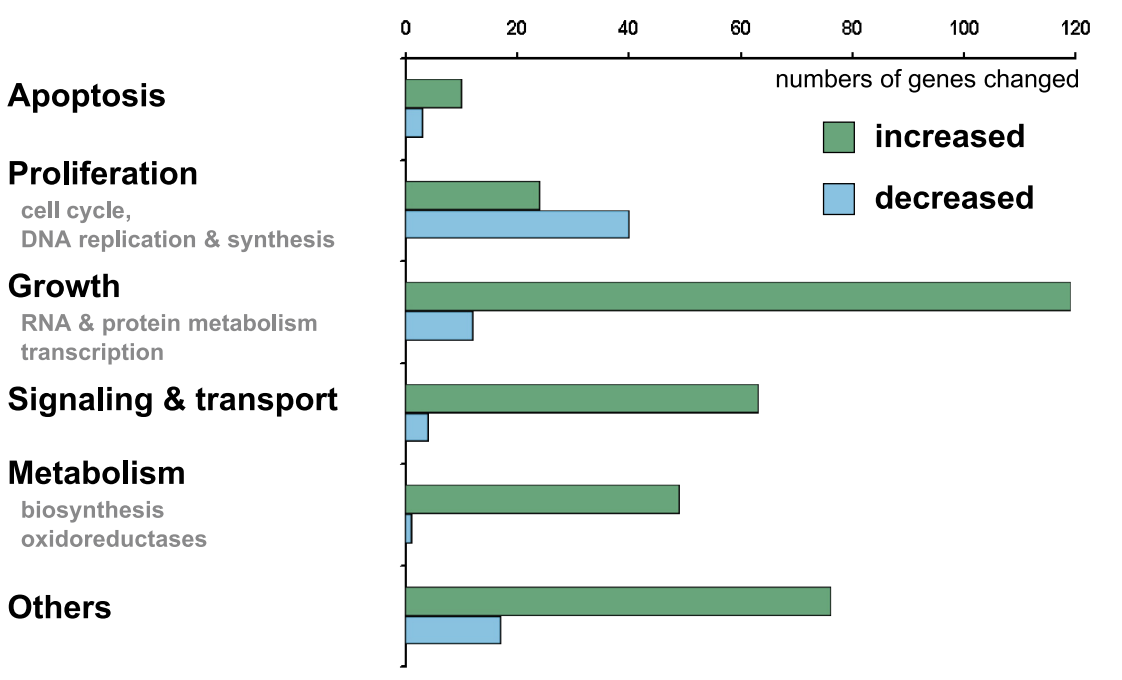

Overall pattern for Cimicifuga is different from that of estradiol and tamoxifen

Fig. 5. Overview of data from a genome-wide expression profiling in MCF-7 cells with black cohosh extract using the Affimetrix Human Genome U133 plus 2.0 array

tion and the active principles are not clear. Estrogen-like, but also selective estrogen receptor modulating activities have been invoked, as well as dopaminergic and serotoninergic properties [23]. Given that steroidal hormone action directly affects the expression of specific genes via their nuclear receptors, we thought that a comparative genome-wide expression profiling study using black cohosh, prototypical estrogen receptor agonist estradiol, and estrogen receptor antagonist tamoxifen, on the estrogen receptorpositive MCF-7 cell line could shed some light in this controversy [24]. Upon treatment of MCF-7 cells with black cohosh extract, a total of 418 genes were up- or downregulated $>1.5$ fold compared to the control. We grouped these genes according to function (Fig. 5). The majority of genes affected were involved with apoptosis, cell proliferation, cell growth, signalling and transport, and with metabolism. For these groups, the genes were mostly up-regulated, with the exception of genes related to cell proliferation, which were predominantly down-regulated. Overall, we observed a regulation of gene expression in a pro-apoptotic manner indicating that the extract may sensitize cells for apoptotic events. The micro-array data were confirmed for selected genes from each group via quantitative real-time PCR. The highly divergent expression patterns found for estradiol and tamoxifen indicated that black cohosh extract has neither estrogen receptor agonistic nor antagonistic properties.

\section{Conclusions and Perspectives}

Pharmaceutical natural products research is presently undergoing a paradigm shift. The challenge of intricate chemistry is ceding its place to an increasingly biology-driven approach. Does pharmaceutical natural product research make sense in an academic setting? History shows that most new compound classes and molecular targets of natural products were found by academic groups. Since 1990, ten new natural product templates have been discovered which have led to compounds currently in clinical trials. Of these ten templates, eight were found by universities and public research organisations [1][3]. Unravelling the biological function of natural products will continue to provide new and unexpected insights into fundamental biological processes, and inspiration for drug discovery. The wealth of new tools and approaches available plays a key role in that process.

Received: December 12, 2005

[1] a) D.J. Newman, G.M. Cragg, K.M. Snader, J. Nat. Prod. 2003, 66, 1022-1037; b) M.S. Butler, Nat. Prod. Rep. 2005, 22, 162-195.

[2] a) R. Breinbauer, I.R. Vetter, H. Waldmann, Angew. Chem. Int. Ed. 2002, 41, 2878-2890; b) T. Henkel, R.M. Brune, H. Müller, F. Reichel, Angew. Chem. Int. Ed. 1999, 38, 643-647.

[3] J. Clardy, C. Walsh, Nature 2004, 432, 829-837. 
[4] E. Ernst, Ann. Int. Med. 2002, 136, 4253.

[5] M. Hamburger, Phytochemistry Rev. 2002, $1,333-344$.

[6] O. Potterat, M. Hamburger, Curr. Org. Chem. 2006, in press.

[7] a) H. Danz, S. Stoyanova, P. Wippich, A. Brattström, M. Hamburger, Planta Med. 2001, 67, 411-416; b) H. Danz, S. Stoyanova, O.A.R. Thomet, H.-U. Simon, G. Dannhardt, H. Ulbrich, M. Hamburger, Planta Med. 2002, 68, 875-880; c) T. Ishihara, K. Kohno, S. Ushio, K. Iwaki, M. Ikeda, M. Kurimoto, Eur. J. Pharmacol. 2000, 407, 197-204.

[8] C. Oberthür, R. Jäggi, M. Hamburger, Fitoterapia 2005, 76, 324-332.

[9] G.-U. Rüster, B. Hoffmann, M. Hamburger, Pharmazie 2004, 59, 236-237.

[10] M.C. Recio, M. Cerdá-Nicolás, O. Potterat, M. Hamburger, J.-L. Rios, Planta Med. 2006, in press.

[11] C. Heinemann, S. Schliemann-Willers, C. Oberthür, M. Hamburger, P. Elsner, Planta Med. 2004, 70, 385-390.

[12] C. Oberthür, C. Heinemann, P. Elsner, E. Benfeldt, M. Hamburger, Planta Med. 2003, 69, 385-389.

[13] C. Oberthür, B. Schneider, H. Graf, M. Hamburger, Chem. Biodiv. 2004, 1, 174182; b) C. Oberthür, M. Hamburger, Planta Med. 2004, 70, 642-645; c) C. Oberthür, H. Graf, M. Hamburger, Phytochemistry 2004, 65, 3261-3268.

[14] K. Schmidt, B. Schubert, B. Huang, S. Stoyanova, M. Hamburger, J. Ethnopharmacol. 2003, 89, 251-260.

[15] a) M. Pattawarapan, K. Burgess, J. Med. Chem. 2003, 46, 5277-5291; b) P. Aebi- scher, J.-L. Ridet, Trends Neurosci. 2001 24, 533-540; c) H.U. Saragovi, K. Gehring, Trends Pharmacol. Sci. 2000, 21, 93-98.

[16] S.J. Pollack, S. J. Harper, Curr. Drug Targets CNS Neurol. Disord. 2002, 1, 59-80.

[17] a) K. Schmidt, W. Günther, S. Stoyanova, B. Schubert, Z. Li, M. Hamburger, Org. Lett. 2002, 4, 197-199; b) K. Schmidt, U. Riese, Z. Li, M. Hamburger, J. Nat. Prod. 2003, 66, 378-383.

[18] a) L.A. Greene, A.S. Tischler, Proc. Natl. Acad. Sci. USA 1976, 73, 2424-2428; b) L.A. Greene, D.R. Kaplan, Curr. Opin. Neurobiol. 1995, 5, 579-587; c) W.J. Friedmann, L.A. Greene, Exp. Cell Res. 1999, 253, 131-142.

[19] a) S. Kida, S.A. Josselin, S.P. de Ortiz, J.H Kogan, I. Chevere, S. Masushige, A.J. Silva, Nat. Neurosci. 2002, 5, 348-355; b) A. Barco, J.M. Alarcon, E.R. Kandel, Cell 2002, 108, 689-703.

[20] U. Riese, E. Ziegler, M. Hamburger, FEBS Lett. 2004, 577, 455-459.

[21] a) A. Butte, Nat. Rev. Drug Discov. 2002, 1, 951-960; b) R.B. Stoughton, S.H. Friend, Nat. Rev. Drug Discov. 2005, 4, 345350.

[22] C.M.H. Watanabe, S. Wolffram, P. Ader P. Rimbach, L. Packer, J.J. Maguire, P.G. Schultz, K. Gohil, Proc. Natl. Acad. Sci. USA 2001, 98, 6577-6580.

[23] V. Viereck, G. Emons, W. Wuttke, Trends Endocrin. Metab. 2005, 16, 214-221.

[24] F. Gaube, L. Pusch, T. Kroll, S. Wölfl, M. Hamburger, Book of Abstracts, 53rd Annual Congress of GA, Florence 2005, p. 104. 